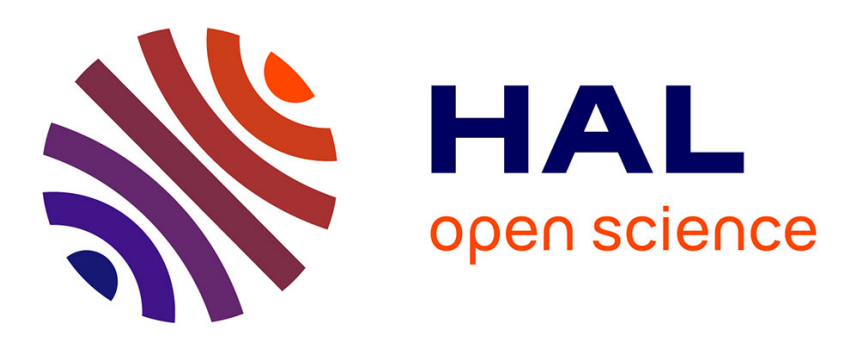

\title{
Les acteurs de l'archéologie préventive et l'UMR 7044 ArcHiMèdE : une collaboration fructueuse
}

\author{
Michel Humm
}

\section{To cite this version:}

Michel Humm. Les acteurs de l'archéologie préventive et l'UMR 7044 ArcHiMèdE : une collaboration fructueuse. Archimède: archéologie et histoire ancienne, 2020, Archimède. Archéologie et histoire ancienne, 8, pp.159-160. 10.47245/archimede.0008.ds3.01 . halshs-03280079

\section{HAL Id: halshs-03280079 \\ https://shs.hal.science/halshs-03280079}

Submitted on 7 Jul 2021

HAL is a multi-disciplinary open access archive for the deposit and dissemination of scientific research documents, whether they are published or not. The documents may come from teaching and research institutions in France or abroad, or from public or private research centers.
L'archive ouverte pluridisciplinaire HAL, est destinée au dépôt et à la diffusion de documents scientifiques de niveau recherche, publiés ou non, émanant des établissements d'enseignement et de recherche français ou étrangers, des laboratoires publics ou privés. 
1 DOSSIER THÉMATIQUE 1

SIUE DEUS SIUE DEA. DÉNOMINATIONS DIVINES DANS LES MONDES GREC ET SÉMITIQUE : UNE APPROCHE PAR LE GENRE

98 DOSSIER THÉMATIQUE 2

TRADITION ET TRANSMISSION DANS L'ANTIQUITÉ : RÉFLEXIONS INTERDISCIPLINAIRES

ACTUALITÉ DE LA RECHERCHE

QUOI DE NEUF Á L'OUEST DE STRASBOURG ? KOENIGSHOFFEN :

ÉTAT DES LIEUX ET DÉCOUVERTES RÉCENTES

159 Michel Humm

Les acteurs de I'archéologie préventive et I'UMR 7044 ArcHiMèdE : une collaboration fructueuse

161 Axelle MURER et Adeline PICHOT

Aux portes de Strasbourg : une occupation de longue durée à Koenigshoffen, en bordure de la route des Romains

180 Marianne BérAud et Adeline PICHOT

Deux nouvelles inscriptions de légionnaires de la légion VIII Augusta

195 VARIA 


\title{
ACTUALITÉ DE LA RECHERCHE
}

\section{«QUOI DE NEUF À L'OUEST DE STRASBOURG ? KOENIGSHOFFEN :} ÉTAT DES LIEUX ET DÉCOUVERTES RÉCENTES »

\author{
dir. Michel HUMM
}

\section{LES ACTEURS DE L'ARCHÉOLOGIE PRÉVENTIVE ET L'UMR 7044 ARCHIMĖDE : UNE COLLABORATION FRUCTUEUSE}

\author{
Michel HUMM \\ Professeur d'histoire romaine \\ Université de Strasbourg \\ michel.humm@unistra.fr
}

\section{RÉSUMÉ}

Les découvertes récentes effectuées le long de la « route des Romains », dans le quartier strasbourgeois de Koenigshoffen, sont issues des nombreux chantiers de fouille d'archéologie préventive organisés à la suite des importants travaux d'aménagement urbain, dont la mise en place d'une ligne de tramway, à l'extrémité orientale de ce quartier. La plupart des acteurs de I'archéologie préventive régionale sont intervenus dans les découvertes réalisées. L'existence de I'UMR 7044 ArcHiMèdE fournit aux archéologues des différents opérateurs, auxquels elle est liée par des conventions de partenariat, la possibilité de confronter et de valoriser leurs découvertes dans le cadre d'un de ses programmes de recherche. Le dossier «Koenigshoffen » ici présenté montre

\begin{tabular}{|c|c|}
\hline & scientifique \\
\hline MOTS-CLÉS & qui découle d'une étroit \\
\hline $\begin{array}{l}\text { Archéologie préventive, } \\
\text { nécropole, } \\
\text { épigraphie. }\end{array}$ & de l'archéologie préventiv \\
\hline
\end{tabular}

The recent discoveries made on route des Romains, in the Strasbourg area of Koenigshoffen, result from the numerous preventive archaeological excavations carried out following major urban development work, including the installation of a tramway line, at the eastern end of the district. Most of the actors of regional preventive archaeology were involved in the discoveries. The research unit UMR 7044 ArcHiMèdE provides the archaeologists working for the various developers, with whom partnership conventions have been signed, with the opportunity to compare and show the value of their discoveries, as part of one of its research programmes. This section focusing on Koenigshoffen shows how fruitful such a close collaboration between actors of preventive archaeology and academia may be.
KeYWORDS

Preventive archaeology, necropolis epigraphy. 
Les fouilles d'archéologie préventive menées depuis plusieurs années dans le quartier strasbourgeois de Koenigshoffen, à I'ouest de Strasbourg, ont permis de révéler une partie importante d'une nécropole du $\mathrm{I}^{\mathrm{er}}$ siècle ap. J.-C. liée à la proximité du camp légionnaire romain d'Argentorate, puis de l'agglomération civile (vicus) qui s'est progressivement développée et organisée le long de la bien nommée « route des Romains » (un axe routier d'origine antique qui reliait le camp romain au Bassin parisien, en passant par le col vosgien de Saverne où se trouvait la statio des Tres Tabernae). Les fouilles récentes ont permis de montrer l'existence d'une occupation de longue durée qui s'étend du début de notre ère à la fin du Moyen Âge, puis des traces d'activités et d'habitats qui reprennent à la fin du XIXe siècle ou au début du $X X^{\mathrm{e}}$ siècle.

Les découvertes récentes sont issues des nombreux chantiers de fouille d'archéologie préventive organisés à la suite des importants travaux d'aménagement urbain, dont la mise en place d'une ligne de tramway, à I'extrémité orientale du quartier de Koenigshoffen. La plupart des acteurs de l'archéologie préventive régionale sont intervenus dans les découvertes réalisées : le Ministère de la Culture, à travers la DRAC Grand Est et le Service régional de I'archéologie, I'INRAP, Archéologie Alsace et ANTEA Archéologie.

L'existence de I'UMR 7044 ArcHiMèdE fournit aux archéologues de toutes ces institutions, liées à elle par des conventions de partenariat, la possibilité de confronter et de valoriser leurs découvertes dans le cadre d'un programme de recherche intitulé « Argentorate, aux origines de Strasbourg » (sous la direction de Pascal Flotté, Archéologie Alsace). Le 8 décembre 2020, P. Flotté, Séverine Blin (CNRS, UMR 8546 AOROC, membre associée de I'UMR 7044 ArcHiMèdE) et Mathias Higelin (Archéologie Alsace, membre de I'UMR ArcHiMèdE) ont organisé une journée d'étude réunissant (par visioconférence) une trentaine de participants, et consacrée à l'architecture funéraire et à l'organisation spatiale de la nécropole de Strasbourg-Koenigshoffen (I ${ }^{e r}$ siècle ap. J.-C. - début du II siècle ap. J.-C.). Cette journée d'étude faisait immédiatement suite à la valorisation d'un tronçon de
I'« allée des tombeaux » découvert aux 8-20 route des Romains, à l'entrée est de Koenigshoffen, au niveau de l'arrêt de tram «Parc des Romains », à laquelle plusieurs des participants ont apporté une contribution directe. Le site, bénéficiant d'un aménagement urbain et pédagogique destiné à présenter les découvertes archéologiques au « grand public », fut officiellement « inauguré » lors d'une cérémonie organisée sur place le 18 septembre 2020, et réunissant les représentants de la Compagnie des Transports Strasbourgeois, de la Ville de Strasbourg, de I'Eurométropole, du Musée archéologique de Strasbourg, de l'Atelier Pandore et des différents opérateurs de l'archéologie préventive. Les travaux issus de la journée d'étude de décembre 2020 feront l'objet d'une publication ultérieure, dans le cadre d'un dossier important consacré à la nécropole de Strasbourg-Koenigshoffen, qui sera publié prochainement par la revue Archimède. Archéologie et histoire ancienne.

Néanmoins, la découverte, en juin 2018, de deux stèles funéraires romaines d'époque flavienne, portant les épitaphes de deux légionnaires de la VIII légion Auguste, premières attestations épigraphiques de la présence de cette unité militaire à StrasbourgArgentorate, justifiait une publication rapide de ces textes inédits ainsi qu'une mise en perspective du contexte archéologique et topographique de leur découverte. À travers ce bref « dossier » consacré aux découvertes les plus récentes effectuées dans la partie la plus orientale de la « route des Romains», à quelques centaines de mètres de l'entrée de l'agglomération de Strasbourg, les deux archéologues d'ANTEA Archéologie responsables de I'opération, Axelle Murer et Adeline Pichot, membres de I'UMR ArcHiMèdE, reviennent sur les circonstances historiques et le contexte topographique de l'occupation de ce site dans la longue durée. De leur côté, Marianne Béraud, épigraphiste et membre associée de I'UMR ArcHiMèdE, et $A$. Pichot publient pour la première fois les textes inédits portés par les deux stèles découvertes. Ce court dossier, qui en annonce d'autres, suffit à montrer la richesse scientifique qui découle d'une étroite collaboration entre les acteurs de l'archéologie préventive et de la recherche universitaire. 